\title{
An evaluation of the stability of image quality parameters of Varian on-board imaging (OBI) and EPID imaging systems
}

\author{
Dennis Nichols Stanley, Niko Papanikolaou, Alonso N Gutierrez \\ University of Texas Health Science Center San Antonio, San Antonio, TX, USA.
}

Received March 19, 2014; Published Online April 08, 2014

[Presented at the Young Investigator's Symposium at the 2014 Annual Meeting of Southwest Chapter of American Association of Physicists in Medicine (AAPM) in San Antonio, Texas, USA]

\section{Conference Proceeding}

\begin{abstract}
Purpose: Quality assurance of the image quality for image guided localization systems is crucial to ensure accurate visualization and localization of target volumes. In this study, the stability of selected image parameters was assessed and evaluated for CBCT mode, planar radiographic $\mathrm{kV}$ mode and the radiographic MV EPID mode.
\end{abstract}

Methods and Materials: The CATPHAN, QckV-1 and QC-3 phantoms were used to evaluate the image quality parameters. The planar radiographic images were analyzed in PIPSpro $^{\mathrm{TM}}$ with spatial resolution $(\mathrm{f} 30, \mathrm{f} 40, \mathrm{f50})$ being recorded. For OBI CBCT, High quality head Full-Fan acquisition and Pelvis Half-Fan acquisition modes were evaluated for Uniformity, Noise, Spatial Resolution, HU constancy and geometric distortion. Dose and $\mathrm{kVp}$ for the OBI were recorded using the Unfors RaySafe Xi system with the $\mathrm{R} / \mathrm{F}$ High Detector for planar $\mathrm{kV}$ and the CT detector for CBCT. Dose for the MV EPID was recorded using a PTW975 Semiflex Ion Chamber, webline electrometer and $1 \mathrm{~cm}$ SolidWater ${ }^{\mathrm{TM}}$.

Results: For each metric, values were normalized to the mean and the standard deviations were recorded. Table 1 shows the standard deviation for all results. Using this, tolerances can be reported as a warning threshold of $1 \sigma$ and an action threshold of $2 \sigma$. Table 2 shows the warning and action tolerances for the planar radiographic modalities while Table 3 and 4 show tolerance levels for the Full-Fan and Half-Fan, respectively.

Conclusion: A study was performed to assess the stability of the basic image quality parameters recommended by TG-142 for the Varian OBI and EPID Imaging systems. The two systems show consistent imaging and dosimetric properties over the evaluated time frame.

Presenting author: Dennis Nichols Stanley; University of Texas

Health Science Center San Antonio, San Antonio, TX, USA.

Cite this article as:

Stanley DN, Papanikolaou N, Gutierrez AN. An evaluation of the stability of image quality parameters of Varian on-board imaging (OBI) and EPID imaging systems. Int J Cancer Ther Oncol 2014; 2(2):020236. DOI: 10.14319/ijcto.0202.36 


\begin{tabular}{|c|c|c|c|c|c|c|c|}
\hline \multicolumn{8}{|c|}{ Table 1: Normalized standard deviations for all evaluated metrics } \\
\hline \multicolumn{8}{|c|}{ Planar Radiographic } \\
\hline \multicolumn{4}{|c|}{$\mathrm{kV}$} & \multicolumn{4}{|c|}{ Mv } \\
\hline$f_{n}$ & 0.015 & $\mathrm{k} \mathrm{V} p_{\mathrm{p}}$ & 0.010 & $f_{n}$ & 0.006 & Dose & 0.005 \\
\hline $\mathrm{f}_{4}$ & 0.008 & Dose & 0.010 & 4 & 0.009 & & \\
\hline$f_{n s}$ & 0.004 & & & $f_{0}$ & 0.018 & & \\
\hline \multicolumn{8}{|c|}{ CECT } \\
\hline \multicolumn{8}{|c|}{ Full.fen CBCT } \\
\hline \multicolumn{2}{|c|}{ Spatial Resolution } & \multicolumn{2}{|c|}{ HU constancy } & \multicolumn{2}{|c|}{ Geometric Distortion } & \multicolumn{2}{|c|}{ Dosimetric } \\
\hline$f_{n t}$ & 0.087 & Lung(PMP) & 0.023 & $A P$ & 0.005 & Dose & \\
\hline fes & 0.086 & Wrter(poly) & 0.065 & LAT & 0.005 & Center & 0.004 \\
\hline$f_{0 s}$ & 0.074 & Bone(Derin) & 0.010 & $\begin{array}{l}\text { Slice thickness } \\
\text { mean }\end{array}$ & 0.056 & Periphery & 0.004 \\
\hline Uniformity & 0.061 & Noise & 0.063 & & & & \\
\hline \multicolumn{8}{|c|}{ Half fan $\mathrm{CBCT}$} \\
\hline \multicolumn{2}{|c|}{ Spatial Resolution } & \multicolumn{2}{|c|}{ HU constancy } & \multicolumn{2}{|c|}{ Geometric Distortion } & \multicolumn{2}{|c|}{ Dosimetric } \\
\hline$f_{9 n}$ & 0.110 & Lung(PMP) & 0.038 & $A P$ & 0.006 & Dose & \\
\hline $\mathrm{f}_{6}$ & 0.116 & Whter(Poly) & 0.058 & LAT & 0.005 & Center & 0.007 \\
\hline $\mathrm{fts}_{\mathrm{s}}$ & 0.173 & Bone(Derlin) & 0.020 & Slice thickness & 0050 & Periphery & 0.003 \\
\hline Uniformity & 0.090 & Noise & 0.079 & mean & 0.037 & & \\
\hline
\end{tabular}

\begin{tabular}{|c|c|c|c|c|c|}
\hline \multicolumn{6}{|c|}{$\begin{array}{l}\text { Table 2: } \\
\text { Thresholds for the Planar radiographic modalities }\end{array}$} \\
\hline \multicolumn{3}{|c|}{$\mathrm{kV}$} & \multicolumn{3}{|c|}{ MV } \\
\hline & Warning & Action & & Warning & Action \\
\hline $\mathbf{f}_{30}$ & $2 \%$ & $4 \%$ & $\mathbf{f}_{30}$ & $2 \%$ & $4 \%$ \\
\hline $\mathbf{f}_{40}$ & $1 \%$ & $3 \%$ & $\mathbf{f}_{40}$ & $1 \%$ & $3 \%$ \\
\hline $\mathbf{f}_{50}$ & $1 \%$ & $3 \%$ & $\mathrm{f}_{50}$ & $1 \%$ & $3 \%$ \\
\hline Dose & $1 \%$ & $2 \%$ & Dose & $1 \%$ & $2 \%$ \\
\hline $\mathbf{k V} \mathbf{p}$ & $1 \%$ & $2 \%$ & & & \\
\hline \multicolumn{6}{|c|}{ Sample size of 30 measurements } \\
\hline
\end{tabular}

\begin{tabular}{|ccc|}
\hline \multicolumn{3}{c}{ Table 4: } \\
Image Quality Thresholds for the \\
Half-Fan CBCT
\end{tabular}

\begin{tabular}{|ccc|}
\hline \multicolumn{3}{c|}{ Table 3: } \\
Image Quality Thresholds for the \\
Full-Fan CBCT \\
\hline Metric & Warning & Action \\
Uniformity & $6 \%$ & $12 \%$ \\
Noise & $6 \%$ & $12 \%$ \\
Spatial Resolution & & \\
$\mathrm{f}_{00}$ & $9 \%$ & $18 \%$ \\
$\mathrm{f}_{40}$ & $9 \%$ & $18 \%$ \\
$\mathrm{f}_{50}$ & $8 \%$ & $16 \%$ \\
HU Constancy & & \\
Lung (PMP) & $3 \%$ & $6 \%$ \\
Water (Poly) & $6 \%$ & $12 \%$ \\
Bone (Derlin) & $1 \%$ & $2 \%$ \\
Geometrical Distortion & $1 \%$ & $2 \%$ \\
AP & $1 \%$ & $2 \%$ \\
L.AT & $6 \%$ & $12 \%$ \\
Slice thickness mean & & \\
Dose $\quad$ Center & $1 \%$ & $2 \%$ \\
Periphery & $1 \%$ & $2 \%$ \\
Sample size of 25 measurements & \\
\hline
\end{tabular}

